\title{
Ichthyosis hystrix of Curth-Macklin
}

INSERM

\section{Source}

INSERM. (1999). Orphanet: an online rare disease and orphan drug data base. Ichthyosis hystrix of Curth-Macklin. ORPHA:79503

Ichthyosis hystrix of Curth-Macklin (IHCM) is a rare type of keratinopathic ichthyosis (see this term) that is characterized by the presence of severe hyperkeratotic lesions and palmoplantar keratoderma (PPK, see this term). 